\title{
Broadly tunable semiconductor lasers using asymmetric dual quantum wells
}

\author{
Ching-Fuh Lin ${ }^{\mathrm{a}, \mathrm{b}, *}$, Bor-Lin Lee ${ }^{\mathrm{a}, \mathrm{b}}$, Miin-Jang Chen ${ }^{\mathrm{a}, \mathrm{b}}$ \\ ${ }^{a}$ Institute of Electro-Optical Engineering, National Taiwan University, Taipei, Taiwan \\ ${ }^{\mathrm{b}}$ Department of Electrical Engineering, National Taiwan University, Taipei, Taiwan
}

Received 11 May 1999; received in revised form 2 September 1999; accepted 3 September 1999

\begin{abstract}
Semiconductor laser with extremely broadband tunability is achieved by engineering gain spectra of the laser material. Using asymmetric dual quantum wells, the gain bandwidth is broadened to about three times wider than that of conventional semiconductor lasers. With such laser material, the single-wavelength operation of the laser is tunable with $90 \mathrm{~nm}$ of tuning range. For dual-wavelength operation, the wavelength separation could be tuned from a few nm to about $55 \mathrm{~nm}$. The two oscillation wavelengths could be almost randomly selected within a spectral range of more than $30 \mathrm{~nm}$. Actively mode-locked operation is also achieved with 10-15 ps pulse widths and a tuning range of 62 nm. (C) 1999 Published by Elsevier Science B.V. All rights reserved.
\end{abstract}

Keywords: Semiconductor lasers; Broadband tunable; Single-wavelength oscillation; Dual-wavelength oscillation; Active mode-locking

\section{Introduction}

Semiconductor lasers have potential applications in many areas due to their compact size, low cost, integration possibility with electronics, and so on. For many applications such as optical communication and spectroscopy, wide-range tunability is important. Conventional semiconductor-laser materials have a gain bandwidth around $50 \mathrm{meV}$, which is the major limitation for tunability. Fortunately, it is possible to engineer the gain spectra of semiconductor lasers. The methods that had been attempted include

${ }^{*}$ Corresponding author. Tel.: + 886-2-2363-5251 ext. 339; fax: +886-2-2363-8247; e-mail: cflin@cc.ee.ntu.edu.tw stacking twin active layers with different bandgaps [1], using $n=1$ and $n=2$ transitions in the same quantum well simultaneously [2], and using multiple quantum wells of different widths [3]. The first method needs a good control on the material gradient during growth, which is not as easy as the thickness control. In the second method the wide spectrum is only obtained for certain device length. The convenience of thickness control for gain-spectrum engineering has thus interested several groups on such exploration recently [4-7]. In this work, we report the use of asymmetric dual quantum wells (ADQWs) to significantly broaden the gain bandwidth. Broadband tunability is achieved for single-wavelength, dual-wavelength operations and active mode-locking. 


\section{Laser material with asymmetric dual quantum wells}

The semiconductor laser material used in this work has two quantum wells that are $40 \AA$ and $75 \AA$ wide respectively. The widths are chosen to make their $n=1$ transition energies separate for $50 \mathrm{meV}$, which is approximately the gain bandwidth for the energy transition in a single QW state for conventional semiconductor laser materials. Fig. 1 shows the calculated transition energy vs. well width for GaAs QW bounded by $\mathrm{Al}_{0.18} \mathrm{Ga}_{0.82}$ As barrier. This figure shows that the $n=1$ transition energies in 40 $\AA$ and $75 \AA$ well separate for about $50 \mathrm{meV}$. Also, the $n=2$ transition energy in $75 \AA$ well separates from the $n=1$ transition energy in $40 \AA$ well for 75 $\mathrm{meV}$. The experiments using slight different spacing of the $n=2$ transition energy in $75 \AA$ and the $n=1$ transition energy in $40 \AA$ had shown no significant influences. Thus, the overall gain bandwidth could be almost three times wider than conventional ones. The width of the $\mathrm{Al}_{0.18} \mathrm{Ga}_{0.82}$ As barrier is $150 \AA$, which is wide enough to ensure no coupling between the two QWs, so the transitions occur at the desired energy levels.

Two types of SLDs had been fabricated on the MOCVD grown substrate with the ADQWs, one with tilted ridge waveguide [8] and the other with bent ridge waveguide [9]. The fabricated SLDs show that their emission spectra are $2 \sim 3$ times wider than conventional SLDs fabricated on substrate with two QWs of the same width [3]. The tilted-strip SLDs

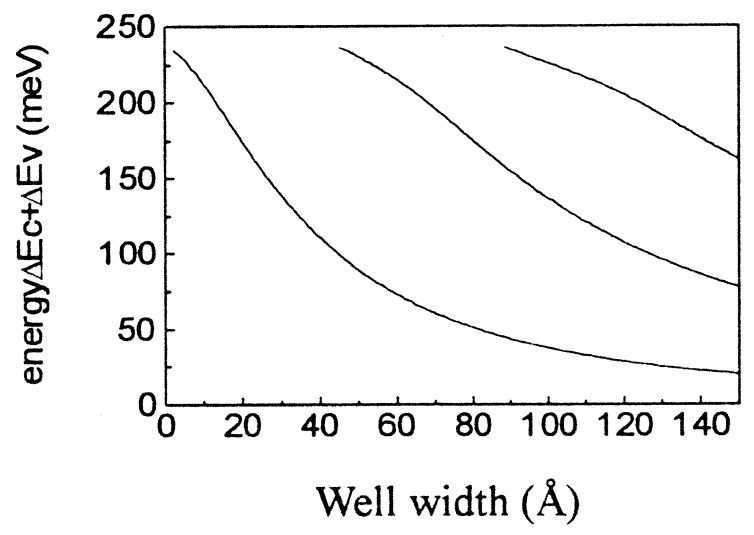

Fig. 1. Calculated transition energy vs. well width for GaAs quantum well bounded by $\mathrm{Al}_{0.18} \mathrm{Ga}_{0.82}$ As barrier. exhibit less ripples due to the Fabry-Perot resonance than the bent-waveguide SLDs because the former ones have both facets with small retro-reflectivity. Therefore, the tilted-stripe SLDs were used in the laser cavity for tuning experiments. The ridge waveguide is tilted at $7^{\circ}$ from the normal of the cleaved facet. The single-wavelength, dual-wavelength, and actively mode-locked operations will be described separately in the following.

\section{Tuning of single-wavelength oscillation}

A ring-cavity configuration schematically shown in Fig. 2 was set up for single-wavelength operation. Two mirrors and one grating were used in the setup. Because the beam emitted from the tilted ridge waveguide has a crescent shape, the triangular ring cavity shown in Fig. 2 has less coupling loss than the usual four-mirror zigzag ring cavity. The ring cavity provides two advantages over the linear cavity. First, because the cross section of the active region is very small and both facets of the SLD have a very small retro-reflectivity, the alignment of the ring cavity is easier than a linear cavity. Second, in the conventional linear cavity, the output beam is accompanied with significant amplified spontaneous emission (ASE) noise. However, in the ring-cavity configuration, one arm of the output beams, beam (a), has very little ASE noise because the ASE noise is first spatially dispersed by the grating and then filtered out by the mirror mount M1. Fig. 3(a) and Fig. 3(b) show the measured spectra emitted from beams (a) and (b), respectively. The ASE suppression ratio in Fig. 3 (a) is significantly improved to $40 \mathrm{~dB}$. This measurement is limited by experimental instruments.

SLDs of different lengths had been used in the cavity. Tuning of wavelength was achieved by rotating the grating angle. The tuning characteristics are shown in Fig. 4(a) and Fig. 4(b). The tuning ranges are 90,70 , and $48 \mathrm{~nm}$ for the 400-, 700-, and $1000-\mu \mathrm{m}$ devices. In the experiment, the operation current was limited to below $400 \mathrm{~mA}$. Further increase of injection current should increase the tuning range, but not much, because the threshold current increases significantly at the two sides of the tuning curve. The ASE suppression ratio of beam (a) was measured to be $40 \mathrm{~dB}$ for the entire tuning range. 
(a) $\lambda_{0}$, Noise free

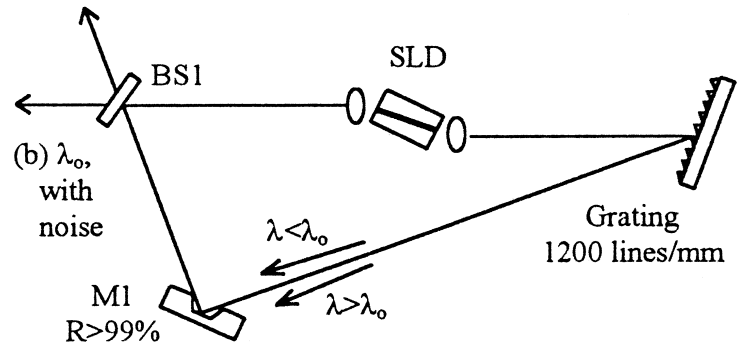

Fig. 2. The experimental setup of ring cavity.

The wide tuning range of using the $400-\mu \mathrm{m}$ SLD indicates that the $n=1$ and $n=2$ transitions in $75 \AA$

(a)

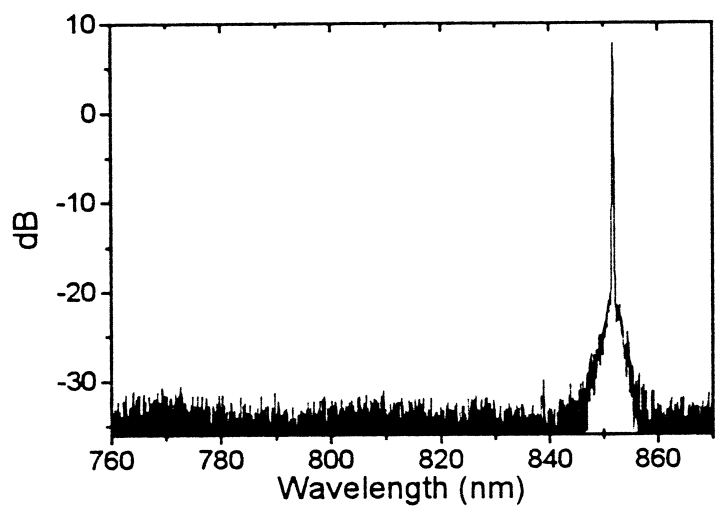

(b)

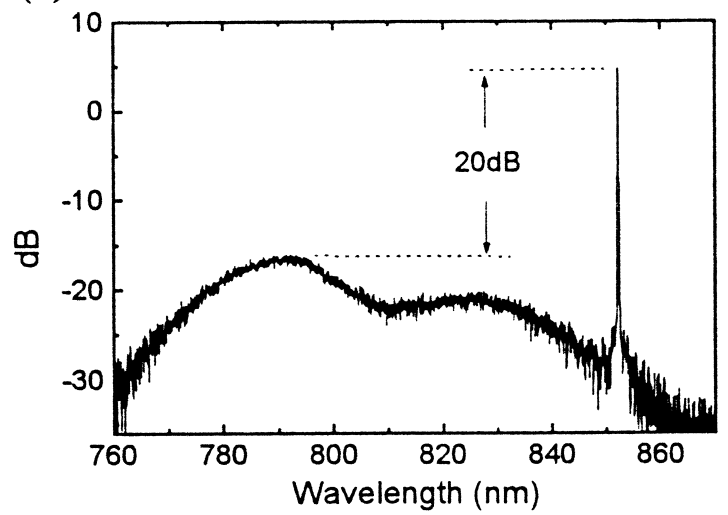

Fig. 3. The spectra measured from beams (a) and (b), respectively, in Fig. 2. (a)

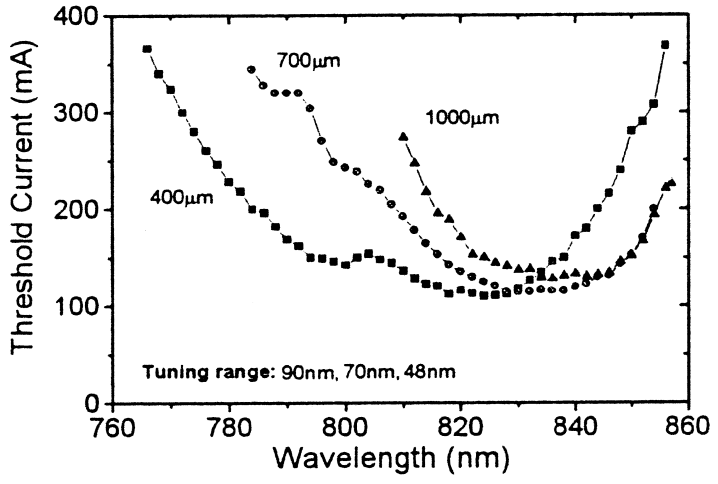

(b)

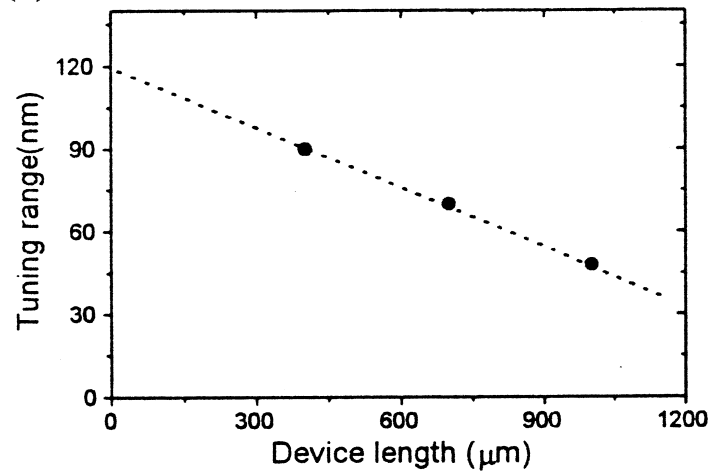

Fig. 4. (a) Threshold current vs. tuning wavelength; and (b) tuning range vs. device length.

well and $\mathrm{n}=1$ transition in $40 \AA$ well could all contribute to the laser oscillation. The length dependence of tuning is caused by two reasons. First, as shown in Fig. 4(a), because the minimum threshold current for using SLDs of different lengths is about the same, the threshold current density for the shorter device is higher, indicating that the shorter SLD is pumped harder. Consequently, band-filling effect leads to a broader spectrum. Second, the exponential growth of amplified spontaneous emission could effectively reduce the emission spectral width of the long SLD, leading to a narrower tuning range. Both factors cause the tuning range to approximately linearly decrease with the device length, as shown in Fig. 4(b). From the extrapolation of the linear curve, 
the ultimate tuning range of very short devices is about $120 \mathrm{~nm}$ for the injection current limited to 400 $\mathrm{mA}$.

\section{Tuning of dual-wavelength oscillation}

For dual-wavelength operation, the cavity schematically shown by Fig. 5 was used. A tilted-strip SLD was also used in this experiment for the reason of reduced Fabry-Perot resonance to enhance the spectral control of the external cavity. A reflectiontype grating telescope [10] was used on one side of the SLD. The grating has 1200 lines /mm. V-shaped double slits were inserted in front of the mirror for the selection of the oscillation wavelengths. An output coupler with $R / T=70 / 30$ was used on the other side of the SLD to form the laser cavity. With the setup, the spectral spacing $(\Delta \lambda)$ of the two wavelengths is related to the slit spacing $(\Delta l)$ simply by the formula: $\Delta \lambda$ (in $\mathrm{nm})=3.83 \Delta l$ (in $\mathrm{mm}$ ). Therefore, moving the $\mathrm{V}$-shaped slits vertically could change the spectral spacing of the two wavelengths. The experiment showed that simultaneous oscillation at two wavelengths could occur with their spacing as far as $55.7 \mathrm{~nm}$. Within this spacing, the two modes could be adjusted to have equal powers. Beyond 55.7 $\mathrm{nm}$, two-mode oscillation is still possible, but the mode of long wavelength always has a larger power. Fig. 6 shows the measured spectra at two spacings: 5 $\mathrm{nm}$ and $55.7 \mathrm{~nm}$.

For two modes with a fixed spectral spacing, they could also be simultaneously tuned by moving the

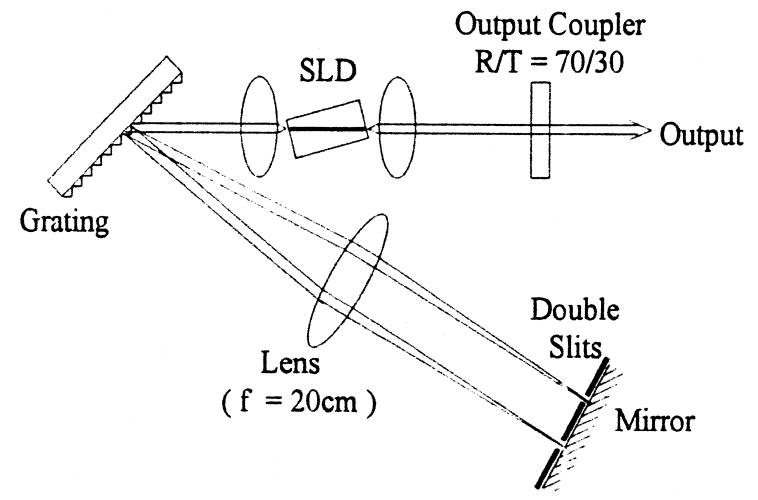

Fig. 5. Schematic of the experimental setup for dual-wavelength operation.

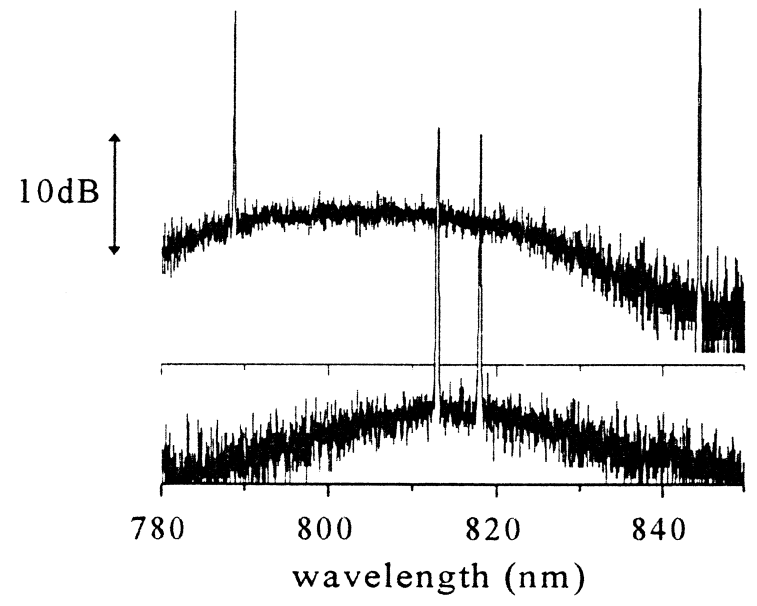

Fig. 6. Measured spectra of two wavelengths at two spacings: 5 $\mathrm{nm}$ and $55.7 \mathrm{~nm}$.

V-shaped slits horizontally. If the two modes are spectrally separated as far as $39 \mathrm{~nm}$, the tuning range is $7 \mathrm{~nm}$. For a narrow spectral spacing like $5.5 \mathrm{~nm}$, the tuning range increases to $34.7 \mathrm{~nm}$. For an even narrower spectral spacing, the tuning range should increase further [11]. Fig. 7 shows the tuning spectra of two wavelengths at fixed spacing. On the other hand, the tuning can also be achieved with one wavelength being varied, while the other is fixed. This is accomplished by using a new type of Vshaped slits. The new type has one vertical side of the $\mathrm{V}$ shape. Vertically moving such $\mathrm{V}$-shaped slits

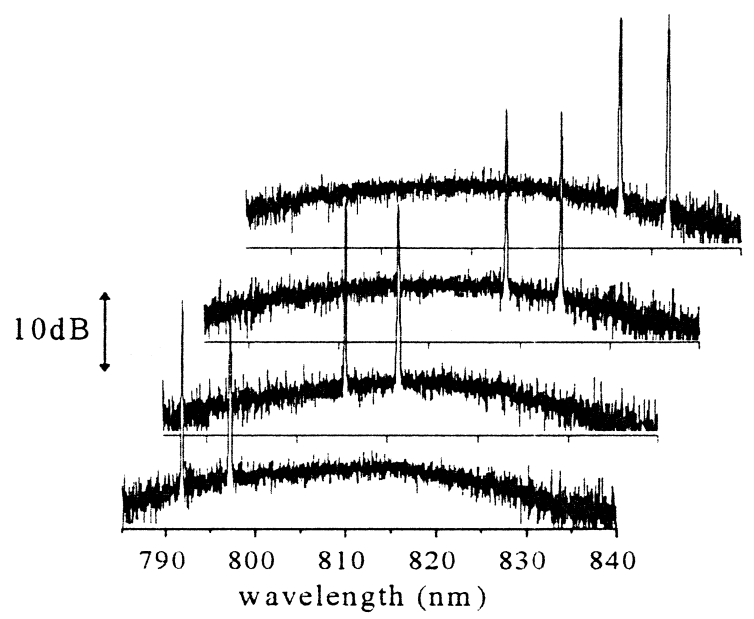

Fig. 7. Tuning spectra of two wavelengths at fixed spacing. 
(a)

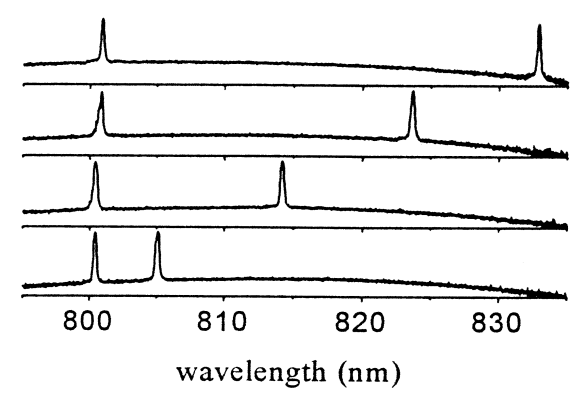

(b)

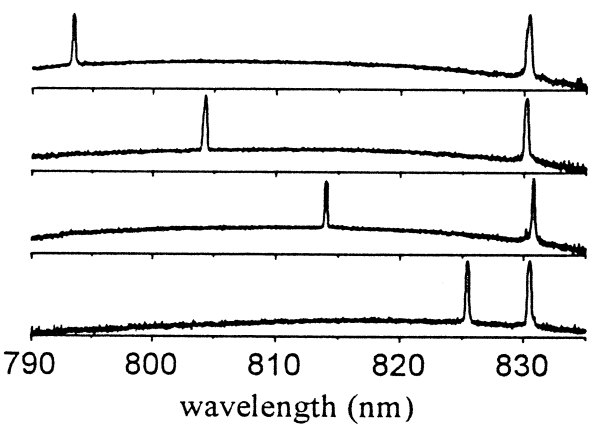

Fig. 8. The tuning spectra for one wavelength varied and the other fixed at: (a) $800 \mathrm{~nm}$; and (b) $830 \mathrm{~nm}$.

could cause one wavelength fixed and the other varied. Fig. 8(a) and Fig. 8(b) show the tuning spectra for one wavelength fixed at $800 \mathrm{~nm}$ and 830 $\mathrm{nm}$, respectively. The tuning of the varied wavelength can be over $30 \mathrm{~nm}$ and the fixed wavelength can be selected between $792 \mathrm{~nm}$ and $835 \mathrm{~nm}$. Those experiments indicate that the two oscillation wavelengths can be almost randomly selected within a spectral range over $30 \mathrm{~nm}$.

Because of the wide tuning range, the two oscillation modes can be contributed either from one QW or two QWs. We had observed in the experiment that the two modes had a strong competition if they were contributed from the same well. In contrast, the two modes had a relatively weak competition if they were contributed from different wells. The reason is because the intraband relaxation in the same well is much faster than the carrier transport between two wells. Consequently, dual-wavelength oscillation is easier to maintain when the two modes are contributed from different wells, respectively. From this point of view, using MQWs of different width for multi-wavelength oscillation should be better than using various quantized levels in the same well.

\section{Tuning of active mode-locking}

In the mode-locking experiment, the ring cavity similar to the one shown in Fig. 2 was used for the advantages mentioned previously. In addition, the pulse train can be more easily synchronized with the RF modulation signal travelling in the ring cavity than in the linear cavity [12]. Fig. 9 shows the measured laser output power vs. the modulation frequency for RF power fixed at $16 \mathrm{dBm}$ and $\mathrm{DC}$ bias at threshold. This frequency response should have already included the effects of the external electronics parasitic and intrinsic carrier transport in SLD. As shown in the figure, the output power is very strong when the laser is modulated at the multiple of the round-trip frequency. In the active mode-locking, modulation at a higher frequency could usually result in shorter mode-locked pulse widths [13]. However, Fig. 9 shows that the response of the laser over the third harmonic is reduced to less than half of that at the round-trip frequency. Therefore, the third harmonic, $\sim 886 \mathrm{MHz}$, of the cavity round-trip frequency was used for mode-locking experiment.

The grating was adjusted to have the laser oscillating near $830 \mathrm{~nm}$. Without applying RF signal,

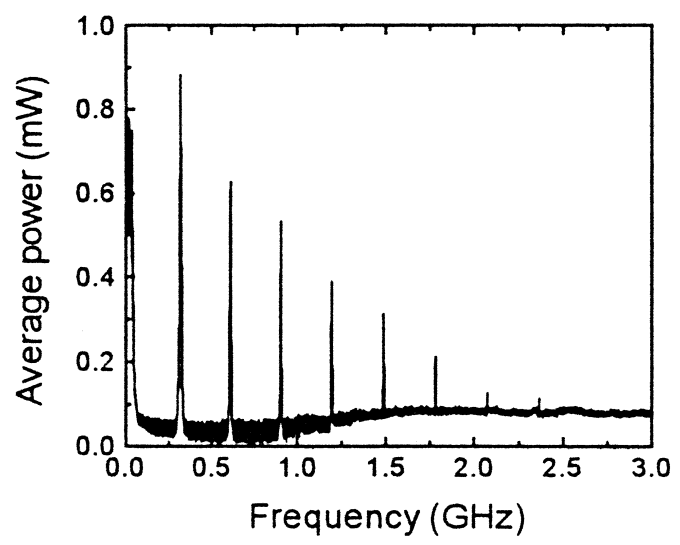

Fig. 9. The measured average power of the laser output (a) in Fig. 2 vs. RF modulation frequency. 
oscillation occurs only with a very narrow spectrum like in the tuning experiment of single wavelength. When the RF modulation is applied, the spectrum is broadened to about 2-4 $\mathrm{A}$ and pulse output could be measured. The experiment shows that the RF modulation frequency has a very significant influence. In order to maintain the measured autocorrelation trace with a well-behaved shape, the RF frequency can be varied only within $0.3 \mathrm{MHz}$, as shown in Fig. 10. On the other hand, the measured autocorrelation shape and width are not strongly affected by the DC biased level as long as it is below the threshold and above $0.6 I_{\text {th }}$. The coincidence of modulation frequency with the cavity round-trip characteristics suggests that the short pulses should be generated by modelocking mechanism instead of gain-switching.

The autocorrelation curves show no secondary pulses resulting from the residual reflectivity of laser-diode facets [13] because the tilted waveguide effectively reduce the retroreflectivity at both facets of the SLD [8]. This autocorrelation trace can be well fitted by the single-sided exponential shape, as shown by the dotted line in Fig. 10. The mode-locking condition is similar for the tuning range from 810 $\mathrm{nm}$ to $857 \mathrm{~nm}$. Fig. 11 shows the lasing threshold, DC bias, and RF modulation frequency vs. tuning wavelength in the experiment. The lasing threshold for $810-857 \mathrm{~nm}$ is approximately between $45 \mathrm{~mA}$ and $70 \mathrm{~mA}$. The RF modulation depth was main-

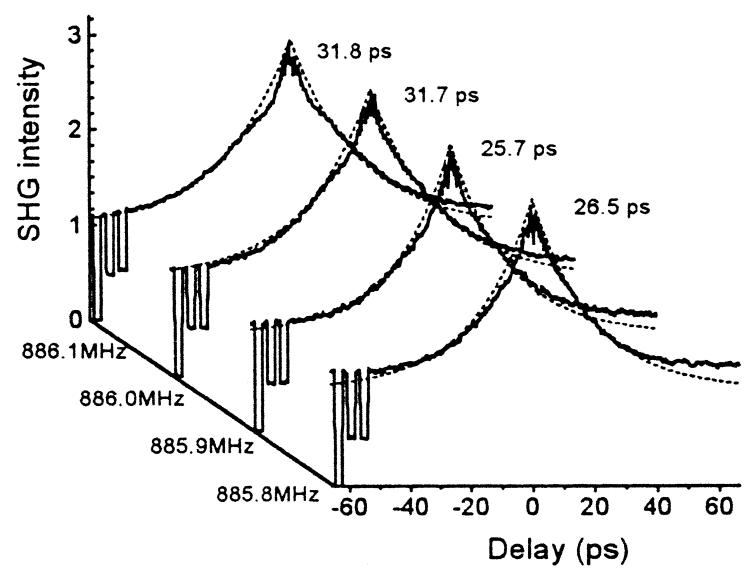

Fig. 10. The autocorrelation trace of the mode-locked pulses at different modulation frequency. (The autocorrelation widths are also indicated and dotted lines are fitted by single-sided exponential functions).

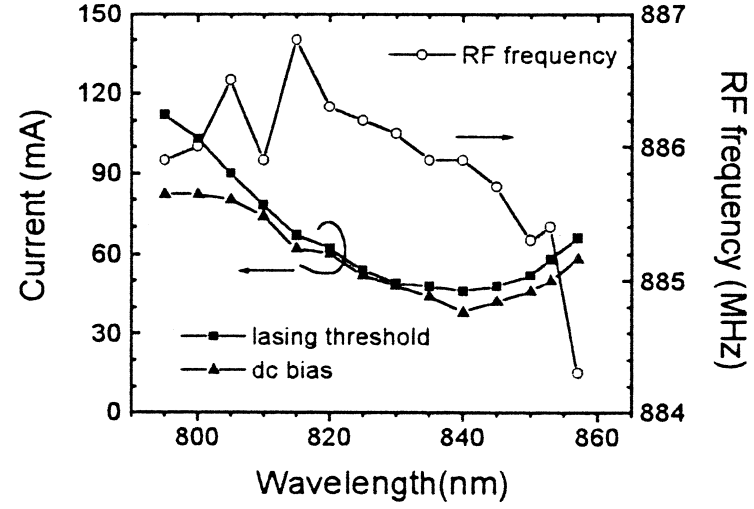

Fig. 11. The threshold current, DC biased current, and RF modulation frequency vs. tuning wavelength for the actively mode-locked lasers in the experiment.

tained at $\sim 0.5 I_{\text {th }}$ for the entire tuning range. For the wavelength below $810 \mathrm{~nm}$, the lasing threshold increases to above $70 \mathrm{~mA}$. The RF modulation depth at $0.5 I_{\text {th }}$ then causes an extra gain for the tail of the pulses, leading to significant broadening of the pulses. The extra gain could be reduced by lowering the DC biased level, so the well-behaved autocorrelation trace was retained by decreasing the DC bias.

Fig. 12 shows the mode-locked pulse width and spectral width vs. the tuning wavelength. The pulse width is calculated by assuming the single-sided exponential pulse shape. The pulse width is $10-15$ ps and in general decreases with decreasing laser wavelength. One reason for the tendency may be due

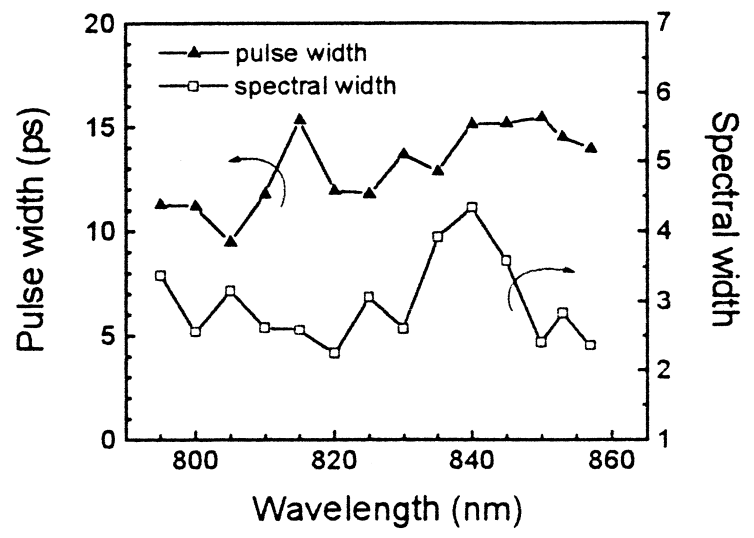

Fig. 12. The mode-locked pulse width and spectral width vs. the tuning wavelength. 
to the increasing differential gain with decreasing wavelength [14]. The corresponding spectrum from beam (a) contains almost no ASE noise, as explained in Section 3. The FWHM of the spectrum is between $2 \AA$ and $4.5 \AA$. The time-bandwidth products of the mode-locked pulses are mostly between one to two. Assuming single-sided exponential pulse shape, the chirped pulses could be possibly compressed to $1-2$ ps. Different from the past observation, [15] there is no evidence of decreased chirp with decreasing lasing wavelength. The reason is probably due to the broad gain bandwidth provided by the asymmetric dual quantum wells.

Although the tuning is demonstrated only for $795-857 \mathrm{~nm}$, a wider tuning range for such actively mode-locked pulses could be expected with proper adjustment of DC bias and RF modulation depth. The wide tuning range is possible due to the broad gain bandwidth provided by the asymmetric dual quantum-well structure. On the other hand, the grating had been replaced with a mirror to see if a broad spectrum could be actively mode-locked to generate extremely short-pulses. The spectral width was then significantly broadened to about $50 \AA$ when the modulation signal was applied. However, no matter what conditions could be possibly adjusted, the auotcorrelation trace shows no well-behaved shapes. The reason is probably because the RF modulation is not strong enough to mode-lock such a broad spectrum.

\section{Conclusion}

In conclusion, the gain bandwidth of semiconductor lasers could be broadened to about three times wider than that of conventional semiconductor lasers using ADQWs. Extremely broadband tunability is therefore possible with such laser material. $90 \mathrm{~nm}$ of tuning range is achieved for single-wavelength operation. For dual-wavelength operation, the spectral spacing of the two modes could be tuned from a few $\mathrm{nm}$ to about $55 \mathrm{~nm}$. Active mode-locking of semiconductor lasers with $\mathrm{ADQWs}$ is also possible to generate pulses of $10-15$ ps with a tuning range of $62 \mathrm{~nm}$.

\section{Acknowledgements}

This work is supported in part by National Science Council, Taipei, Taiwan, ROC, under the contract No. NSC88-2215-E-002-021 and No. NSC2112-M-002-038.

\section{References}

[1] O. Milkami, H. Yasaka, N. Noguchi, Appl. Phys. Lett. 56 (1990) 987.

[2] A.T. Semenov, V.R. Shidlovski, S.A. Safin, Electron. Lett. 29 (1993) 854.

[3] C.-F. Lin, B.-L. Lee, P.-C. Lin, IEEE Photon. Technol. Lett. 8 (1996) 1456.

[4] C.-F. Lin, B.-L. Lee, Appl. Phys. Lett. 71 (1997) 1598.

[5] H.S. Gingrich, D.R. Chumney, S.-Z. Sun, S.D. Hersee, L.F. Lester, S.R.J. Brueck, IEEE Photon. Technol. Lett. 9 (1997) 155.

[6] X. Zhu, D.T. Cassidy, M.J. Hamp, D.A. Thompson, B.J. Robinson, Q.C. Zhao, M. Davies, IEEE Photon. Technol. Lett. 9 (1997) 1202.

[7] T.F. Krauss, G. Hondromitros, B. Vogele, R.M. De La Rue, Electron. Lett. 33 (1997) 1142.

[8] G.A. Alphonse, D.B. Gilbert, M.G. Harvey, M. Ettenberg, IEEE J. Quantum Electron. 24 (1988) 2454.

[9] C.-F. Lin, C.-S. Juang, IEEE Photon. Technol. Lett. 8 (1996) 206.

[10] C.-L. Wang, C.-L. Pan, Appl. Phys. Lett. 64 (1994) 3089.

[11] K.-S. Lee, C. Shu, IEEE J. Quantum Electron. 33 (1997) 1832.

[12] N.A. Olsson, C.L. Tang, IEEE J. Quantum Electron. 17 (1981) 1977.

[13] J.E. Bowers, P.A. Morton, A. Mar, S.W. Corzine, IEEE J. Quantum Electron. 25 (1989) 1426.

[14] M. Hofmann, M. Koch, J. Feldmann, W. Elsaber, E.O. Gobel, W.W. Chow, S.W. Koch, IEEE J. Quantum Electron. 30 (1994) 1756.

[15] M. Serenyi, J. Kuhl, E.O. Gobel, Appl. Phys. Lett. 50 (1987) 1213. 